Correction to Met. Trans. A, 1979, vol, $10 \mathrm{~A}$

Critical Fracture Stress and Fracture Strain Models for the Prediction of Lower and Upper Shelf Toughness in Nuclear Pressure Vessel Steels by R. O. Ritchie, W. L. Server, and R. A. Wullaert

\title{
Page 1558
}

Eq. [1] should read:

$$
\frac{\sigma_{y y}}{\sigma_{o}}=f(N)\left[\frac{J}{\sigma_{O} \epsilon_{O} I x}\right]^{1 / N+1}
$$

such that $\frac{\sigma_{y y}}{\sigma_{y}}=f(N)\left[\frac{\left(1-\nu^{2}\right)}{I}\right]^{1 / N+1}\left[\frac{x}{\left(K / \sigma_{y}\right)^{2}}\right]^{-1 / N+1}$

Thus, applying the RKR criterion

$$
\begin{aligned}
& K_{I c}=\beta^{-[(N+1) / 2]} l_{o}^{*}{ }^{1 / 2}\left[\frac{\sigma_{f}^{*}[(N+1) / 2]}{\sigma_{y}^{[(N-1) / 2]}}\right] \\
& \text { where } \beta=f(N)\left[\frac{\left(1-\nu^{2}\right)}{I}\right]^{1 / N+1}
\end{aligned}
$$

Table I then becomes:

\begin{tabular}{lccc}
\hline$N$ & $I$ & $f(N)$ & $\beta^{1}$ \\
\hline$N=3$ & 551 & 1.94 & 1.23 \\
$N=5$ & 5.02 & 2.22 & 1.66 \\
$N=9$ & 4.60 & 2.46 & 209 \\
$N=13$ & 4.40 & 2.58 & 230 \\
$N=25$ & 4.14 & 2.73 & 2.57 \\
$N=49$ & 3.96 & 2.83 & 2.75 \\
$N=99$ & 3.84 & 2.90 & 2.86 \\
\hline \multicolumn{1}{l}{${ }^{1}$ Computed for $\nu=033$, typical of steel. } \\
\hline
\end{tabular}

Correction to Met. Trans. A, 1980, vol. 11A

Low Stress and Superplastic Creep Behavior of Zn-22 Pct Al Eutectoid Alloy by A. Arieli, A. K. S. Yu, and A. K. Mukherjee

\section{Page 188}

The published Fig. 14 and accompanying caption should read Fig. 15.

\section{Page 189}

The published Fig. 15 and accompanying caption should read Fig. 14. 